\title{
Photoemission time versus streaking delay in attosecond time-resolved solid state photo- emission
}

\author{
Andreas Gebauer ${ }^{1,2^{*}}$, Sergej $\mathrm{Neb}^{1}$, Walter Enns ${ }^{1}$, Ulrich Heinzmann ${ }^{1}$, Andrey K. \\ Kazansky ${ }^{3,4,5}$, and Walter Pfeiffer ${ }^{1}$ \\ ${ }^{1}$ Bielefeld University, Universitätsstr. 25, 33615 Bielefeld, Germany \\ ${ }^{2}$ University of Kaiserslautern, Erwin Schrödinger Str. 46, 67663 Kaiserslautern, Germany \\ ${ }^{3}$ University of the Basque Country, 20080 San Sebastián, Spain \\ ${ }^{4}$ Donostia International Physics Center (DIPC), Paseo Manuel de Lardizabal 4, 20018 San Sebastián, \\ Spain \\ ${ }^{5}$ IKERBASQUE, Basque Foundation for Science, Maria Diaz de Haro 3, 48013 Bilbao, Spain
}

\begin{abstract}
Time-dependent Schrödinger equation simulations for a onedimensional model potential reveal that the delay extracted from a streaking spectrogram does not reflect the photoemission time if the streaking field inside the solid cannot be neglected.
\end{abstract}

\section{Introduction}

The availability of attosecond extreme ultraviolet (EUV) pulses paved the way to observe electron dynamics at atomic scales at solid surfaces on their natural time scales [1]. In streaking spectroscopy the interaction of a photoelectron with an intense near-infrared (IR) streaking field reveals relative delays of different emission channels with up to 10as resolution [2]. Improvements of the temporal resolution showed the impact of various effects on the observed relative photoemission delays. In addition to propagation time differences due to the electron mean free path (MFP) and differences in kinetic energy [13], it was shown that the band structure [4-6], electron-hole interaction and intra-atomic many-body effects need to be taken into account [2].

In attosecond time-resolved streaking of EUV photoemission from solids the interaction of the emitted photoelectron with a few-cycle IR pulse allows probing the photoemission dynamics. For particular refractive indices and illumination geometries the component perpendicular to the surface of the IR field inside the material is negligible. Under these conditions the delay extracted from the streaking spectrogram indeed reflects the photoemission time at which the photoelectron crosses the bulk-vacuum interface, which can be envisioned as a common finish line for different photoemission channels. Hence the notion of a "stopwatch" positioned at the interface is applicable and provides an intuitive interpretation of the measured relative streaking delays. In the more general case when there is a non-negligible streaking field inside the solid this simple picture fails, as it is

Corresponding author: agebauer@physik.uni-bielefeld.de 
demonstrated here. Note that attosecond streaking spectroscopy employing EUV photon energies in the range of several tens to hundred $\mathrm{eV}$ probes only the topmost few $\AA$ of the surface. The actual streaking field distribution at the interface at these scales is unknown since the Fresnel picture, i.e. the abrupt transition between bulk and vacuum, fails. So in general the impact of an inhomogeneous streaking field distribution on the streaking process is relevant and needs careful consideration.

Here we solve the one-dimensional time-dependent Schrödinger equation (TDSE) in a single-electron approach [7] for model cases that take an inhomogeneous streaking field distribution into account. Although streaking field penetration is included in some theoretical models $[2,7]$, the consequences for the interpretation of streaking delays is rarely discussed. It turns out that a penetrating streaking field significantly influences the streaking delay although the photoemission time remains almost identical.

\section{Results and discussion}

To demonstrate the effect of a penetrating streaking field we choose a hypothetical model system (jellium solid, internal potential $9.5 \mathrm{eV}, 91 \mathrm{eV}$ EUV photon energy) with infinite mean free path. This allows demonstrating the effect based on well-defined wave packet trajectories. Figure $1 \mathrm{~b}$ shows the position expectation value of a photoelectron wave packet generated from a core level $\left(\mathrm{E}_{0}=-30.4 \mathrm{eV}\right)$ localized at $-32.6 \AA$. During the excitation the position expectation value deviates from the classically expected behavior since there is no well-defined birth time of the photoelectron. For a photoelectron origin closer to the surface this interferes with the definition of an arrival time of the photoelectron at the interface. This is avoided by setting the origin to $-32.6 \AA$ and assuming an infinite mean free path. As indicated in Fig. 1a we consider two cases for the streaking field distribution, i.e. a nonpenetrating streaking field (blue dashed line) and the case with internal streaking field (red

(a)

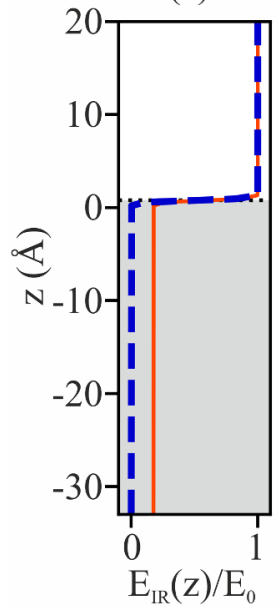

(b)

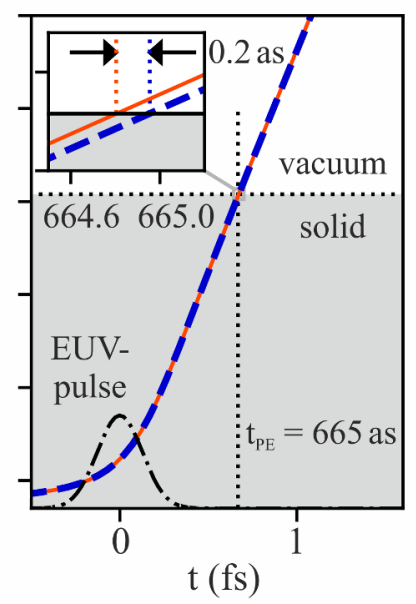

(c)

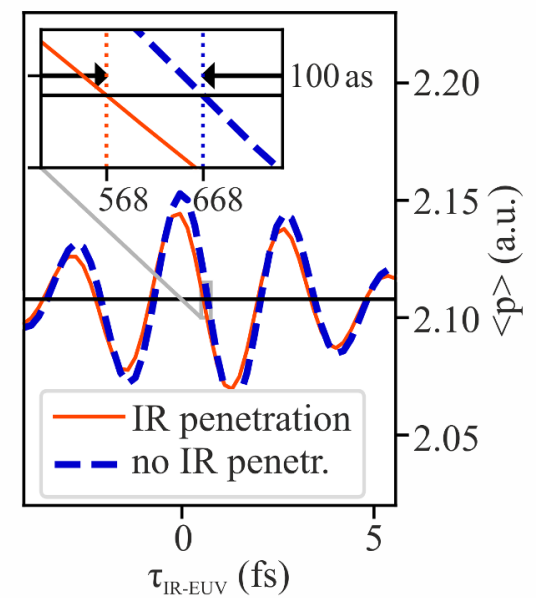

Fig. 1. (a): z-component of the inhomogeneous electric field of the IR pulse in z-direction. Blue dashed lines mark the case without streaking field penetration and red solid lines mark the case with $18 \%$ IR field amplitude penetration. (b): Transient position expectation value of a photoelectron wave packet generated at $-32.6 \AA$ by a 300 as EUV pulse (black dash-dotted line). The intersection of $\langle\mathrm{z}\rangle$ with the surface (horizontal black dotted line at $\mathrm{z}_{\mathrm{s}}=0.78 \AA$ ) corresponds to the propagation time of the wave packet to the surface $t_{\mathrm{PE}}$ (vertical black dotted line). The inset shows $\langle z\rangle$ close to the surface. (c): Corresponding momentum expectation values extracted from streaking spectrograms for penetrating and non-penetrating streaking fields. The absolute delay deviation is shown in the inset. The horizontal black solid line marks $\langle p\rangle$ without IR interaction. 
solid line). The trajectories for both cases differ only slightly (inset of Fig. 1b). The photoelectron wave packet that is exposed to an internal streaking field reaches the surface only about 0.2 as earlier than the one that experiences no streaking field inside the material.

Based on these almost identical trajectories one might expect also marginally different streaking spectra. However, this is not observed. Figure 1c displays the streaking spectra, i.e. the variation of the momentum expectation value for the wave packet as a function of the relative delay $\tau_{\text {IR-EUV }}$ between EUV excitation and the IR streaking field. The momentum distribution is recorded well after the IR streaking field has vanished and the electron wave packet has left the interface region. For a non-penetrating streaking field the determined absolute streaking delay as obtained from the calculated streaking spectrogram is $\tau_{0}=667.6$ as. This value agrees well with the solid-vacuum interface crossing time $\mathrm{t}_{\mathrm{PE}}=665.0$ as, confirming the applicability of the "stopwatch" picture for a vanishing streaking field inside the solid. In contrast, for the penetrating streaking field a streaking delay $\tau_{0}=567.7$ as is obtained, i.e. 100 as smaller than for the case of a non-penetrating streaking field. So in this case the delay extracted from the streaking measurement does not reflect the solid-vacuum interface crossing time, i.e. the photoemission time. Seemingly, a very subtle modification in the momentum distribution induced by the penetrating streaking field has a significant effect on the streaking spectrogram. As a consequence, an intuitive interpretation of attosecond streaking delays based on an "attosecond-stopwatch" fails as soon as the streaking field penetration can no longer be neglected.

In the above discussed example an origin deep inside the solid was chosen for demonstration purpose. The effect is also relevant for a realistic MFP and an effective emission origin close to the surface. The simulation for such conditions and two different core levels reveals relative streaking delay differences between the penetrating and nonpenetrating IR field case in the order of 10 as. This relative delay is in the same order of magnitude as typical propagation or intra-atomic effects [2]. Note that the impact of the penetrating IR field was taken into account in the study identifying the intra-atomic delays [2]. Summarizing the present study, photoemission time and streaking delays directly correspond to each other only for negligible internal streaking fields.

\section{References}

1. A. L. Cavalieri, N. Müller, T. Uphues, V. S. Yakovlev, A. Baltuška, B. Horvath, B. Schmidt, L. Blümel, R. Holzwarth, S. Hendel, M. Drescher, U. Kleineberg, P. M. Echenique, R. Kienberger, F. Krausz, U. Heinzmann, Nature 449, 1029 (2007)

2. F. Siek, S. Neb, P. Bartz, M. Hensen, C. Strüber, S. Fiechter, M. Torrent-Sucarrat, V. M. Silkin, E. E. Krasovskii, N. M. Kabachnik, S. Fritzsche, R. Díez Muiño, P. M. Echenique, A. K. Kazansky, N. Müller, W. Pfeiffer, U. Heinzmann, Science 357, 1274 (2017)

3. S. Neppl, R. Ernstorfer, A. L. Cavalieri, C. Lemell, G. Wachter, E. Magerl, E. M. Bothschafter, M. Jobst, M. Hofstetter, U. Kleineberg, J. V. Barth, D. Menzel, J. Burgdörfer, P. Feulner, F. Krausz, R. Kienberger, Nature 517, 342 (2015)

4. A. G. Borisov, D. Sánchez-Portal, A. K. Kazansky, P. M. Echenique, Phys. Rev. B 87, 121110 (2013)

5. Z. Tao, C. Chen, T. Szilvási, M. Keller, M. Mavrikakis, H. Kapteyn, M. Murnane, Science 353, 62 (2016)

6. L. Kasmi, M. Lucchini, L. Castiglioni, P. Kliuiev, J. Osterwalder, M. Hengsberger, L. Gallmann, P. Krüger, U. Keller, Optica 4, 1492 (2017)

7. A. K. Kazansky, P. M. Echenique, Phys. Rev. Lett. 102, 177401 (2009) 